\title{
Two-step full-thickness resection with anchor device of a laterally spreading tumor inside a rectal recess in a patient with an ileorectal anastomosis
}

For a recurrent adenoma or adenocarcinoma that has arisen on an anastomotic suture, surgical management with "redo" anastomosis is usually considered the standard treatment. The reasons for this are the high risk of recurrence or incomplete excision after endoscopic mucosal resection (EMR) and the extreme difficulty of endoscopic submucosal dissection (ESD) owing to scar tissue. Few data have been published in the literature about the use of full-thickness resection device (FTRD)-assisted treatment of perianastomotic laterally spreading tumors. We describe the case of 70-year-old woman who had undergone ileorectal anastomosis following colectomy and lower anterior resection for synchronous adenocarcinomas of the right colon and proximal rectum. The patient underwent a follow-up lower gastrointestinal (GI) endoscopy, which showed a granulartype laterally spreading tumor (LST-G) of $3 \mathrm{~cm}$, which was completely involving a recess of the remnant rectum and the suture line. Because of the difficulty of this case, we decided, during a multidisciplinary case discussion, to perform resection using an FTRD.

The procedure was performed using an FTRD with the addition of an anchor device (Ovesco, Tübingen, Germany) ( Video 1). Histology showed a tubulovillous adenoma with low grade dysplasia. After 3 months, lower GI endoscopy revealed a recurrence of granular adenomatous tissue over the clip. A hot-snare procedure plus argon plasma coagulation (APC) was performed to completely resect the lesion. Histology again showed a tubulovillous adenoma. After a further 3 months, lower Gl endoscopy showed complete eradication of the lesion, with the clip no longer being present.

Even if not curative with the initial procedure, treatment of perianastomotic lesions with an FTRD could represent a safe and effective strategy, particularly in the

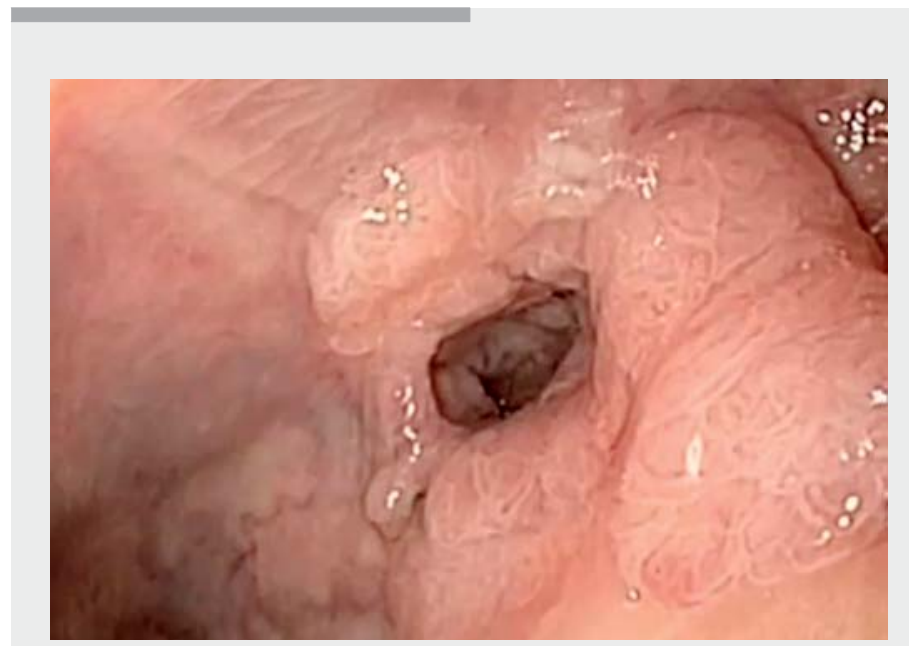

$\checkmark$ Video 1 Full-thickness resection of a granular-type laterally spreading tumor of $3 \mathrm{~cm}$ that was completely involving a recess of the remnant rectum and the suture line using a full-thickness resection device. A recurrence of the lesion was subsequently treated with hot-snaring plus argon plasma coagulation.

setting of former surgical patients, to avoid destructive additional surgery and, in such patients, the need for a permanent stoma. There are no data currently in the literature about the use of an FTRD in a colonic or rectal remnant recess.

Endoscopy_UCTN_Code_TTT_1AQ_2AC

Competing interests

The authors declare that they have no conflict of interest.

The authors

Benedetto Mangiavillano ${ }^{1,2}$, Francesco

Auriemma ${ }^{1}$, Mario Bianchetti ${ }^{1}$, Alessandro Repici ${ }^{2,3}$

1 Gastrointestinal Endoscopy Unit, Humanitas Mater Domini, Castellanza (VA), Italy

2 Humanitas University, Milan, Italy

3 Digestive Endoscopy Unit, Humanitas Research Hospital, Milan, Italy
Corresponding author

\section{Benedetto Mangiavillano, MD}

Gastrointestinal Endoscopy Unit, Humanitas

- Mater Domini, Via Gerenzano n.2,

21053 - Castellanza (VA), Italy

bennymangiavillano@gmail.com

Bibliography

DOI https://doi.org/10.1055/a-1108-1369

Published online: 12.2 .2020

Endoscopy 2020; 52: E286

(c) Georg Thieme Verlag KG

Stuttgart · New York

ISSN 0013-726X 